\title{
Thesen zur Regulierung der dualen Rundfunkordnung*
}

\author{
Wolfgang Hoffmann-Riem
}

Die duale Rundfunkordnung hat sich in Deutschland bewährt. Ibre Regulierung muss aber auf die Veränderungen der Multimedia-Märkte abgestimmt sein. Die zukünftige Medienregulierung wird den der Veranstaltung vor-, neben- und nachgelagerten Bereichen vermebrte Aufmerksamkeit zuwenden müssen; vor allem ist für die Verwirklichung von Zugangschancengerechtigkeit zu sorgen. Selbstregulierung ist für die Medien unabdingbar, sie bedarf aber ergänzender hobeitlicher Sicherungen der Funktionsfähigkeit der Medienordnung.

\section{Die Grundidee der dualen Rundfunkordnung}

1. Die Einführung einer dualen Rundfunkordnung in Deutschland war nicht die Umsetzung einer systematisch entwickelten Konzeption; diese Rundfunkordnung hat sich vielmehr in verschiedenen Schritten in einem Feld entwickelt, das durch neue technologische Möglichkeiten, publizistische Entfaltungs- und ökonomische Verwertungsinteressen, politische Konflikte, rechtliche Vorgaben und verfassungsgerichtliche Interventionen geprägt war.

2. Die duale Rundfunkordnung baut auf je unterschiedlichen Strukturen des privatwirtschaftlichen Rundfunks einerseits und des öffentlich-rechtlichen Rundfunks andererseits auf. Die Kombination beider Säulen soll die Rundfunkfreiheit dadurch fördern, dass es in den Strukturen verankerte unterschiedliche Programmorientierungen und zugleich Möglichkeiten der Kompensation der Nachteile eines Systems durch Vorteile des anderen und umgekehrt gibt (Idee struktureller Diversifikation). Der bedeutsamste Strukturunterschied besteht in dem zwischen Privatwirtschaftlichkeit einerseits und Gemeinwirtschaftlichkeit andererseits. Beide Säulen stehen im Wettbewerb zueinander. In programmlicher Hinsicht ist dies ein publizistischer Wettbewerb, kein ökonomischer.

3. Das Bundesverfassungsgericht sieht in der Verkoppelung beider Säulen eine Rechtfertigung dafür, dass die Vielfaltsanforderungen für privatwirtschaftlichen Rundfunk verringert werden können, aber nur „solange und soweit die Wahrnehmung dieser Aufgaben jedenfalls durch den öffentlich-rechtlichen Rundfunk wirksam sichergestellt ist“. Die duale Rundfunkordnung ist zwar nicht verfassungsrechtlich aufgegeben. Die Beseitigung der Dualität hätte aber erhebliche normative Folgerungen für die Anforderungen an die Rundfunkordnung, etwa an die Betätigung von privaten Rundfunkveranstaltern.

\section{Veränderungen der Ausgangslage}

4. Die Europäisierung des Rundfunkwesens hat die Entstehung einer dualen Rundfunkordnung befördert. Die EG-Aktivitäten zielen durchgängig auf eine Stärkung der pri-

* Zusammenfassende Thesen der Untersuchung „Regulierung der dualen Rundfunkordnung Grundsatzfragen“, die in Kürze als Monografie im Nomos-Verlag erscheint. 
vatwirtschaftlichen Säule der Rundfunkordnung, stellen aber die duale Ordnung nicht grundsätzlich in Frage. Durch das auf den öffentlich-rechtlichen Rundfunk bezogene Protokoll zum Amsterdamer Vertrag ist die Dualität bestärkt worden.

5. Neuerungen bei den Informationstechnologien, den Netzinfrastrukturen, den Geräten sowie den technischen und inhaltlichen Kommunikationsdiensten führen zur Vervielfältigung der Übertragungskapazitäten, zu neuen Inhalten der Individual- und Massenkommunikation, zu neuen Arten und Pfaden der Verbreitung und zu neuen Programm- und Vermarktungsformen, aber auch zu neuen Rezeptionsmöglichkeiten und -gewohnheiten. Die Informationstechnologie-, Telekommunikations- und Medienmärkte durchdringen und überlagern einander zunehmend (Konvergenzen). Neue Anbieter treten auf und alte wie neue Akteure versuchen, ihre Aktivitäten auf unterschiedliche Glieder der Multimedia-Wertschöpfungskette zu erstrecken bzw. an mehreren Stellen in dem sich entwickelnden Multimedia-Wertschöpfungsnetzwerk zu handeln, dabei insbesondere Größen- und sonstige Synergieeffekte zu nutzen.

6. Die früher als relativ einheitlicher Vorgang gehandhabte Rundfunkveranstaltung i. w. S. (Einheit von Produktion, Veranstaltung und Verbreitung) löst sich zunehmend in einzelne Segmente auf und wird zugleich durch neuartige Handlungssegmente ergänzt. Das integrierte, veranstalterzentrierte Rundfunksystem wandelt sich zu einem desintegrierten System von Dienstleistern verschiedener Elemente der (Massen)kommunikation. Die Rundfunkordnung ist nur noch ein Teil einer übergreifenden, dienstebezogen diversifizierten Kommunikations- und Informationsordnung, deren Entwicklungsstand auf die Möglichkeit der Ausgestaltung der speziellen Rundfunkordnung zurückwirkt.

7. Rundfunkveranstaltung i. e. S. - verstanden insbesondere als Festlegung der Struktur des Programms, Zusammenstellung der Segmente und Angebot unter einheitlichem Namen - lässt sich zwar rechtlich von den ihr vor-, neben- und nachgelagerten Bereichen trennen. Allein auf die Veranstaltung i. e. S. zu sehen und Regulierung nur darauf auszurichten, geht aber angesichts der Überlappungen und Vernetzungen sowie wechselseitigen Austauschbarkeiten an der Realität der Medienmärkte vorbei. Der Grundstein für Gefährdungen einer funktionsfähigen Medienordnung kann in den Bereichen der Programmproduktion, -verbreitung und -rezeption ebenso gelegt werden wie bei der Rundfunkveranstaltung i. e. S. Neue Risiken für die Funktionsfähigkeit der Medienordnung können insbesondere durch diverse Zugangsfilter (s. u. Punkt 18 ff.) verwirklicht werden.

8. Die Entkoppelung und Vermehrung der für die Rundfunkfreiheit erheblichen Segmente und Ebenen und deren notwendiges Zusammenwirken führen zu neuen rundfunkverfassungsrechtlichen Fragen und zu neuen Aufgaben der Medienregulierung.

\section{Die Berechtigung hoheitlicher Rundfunkregulierung}

9. Die Berechtigung hoheitlicher Rundfunkregulierung entfällt durch die schon weitgehend erfolgte und weiter voranschreitende Überwindung der Knappheit von Übertragungskapazitäten nicht notwendig. Die Frequenzzuteilung unter Knappheitsbedingungen bot sich lange Zeit als rechtstechnischer Anknüpfungspunkt einer die fernmelderechtliche Regelung ergänzenden rundfunkrechtlichen Regulierung an; bei Wegfall der Knappheit können andere Anknüpfungspunkte genutzt werden. 
10. Rundfunkrechtliche Regulierung folgte stets eigenständigen Zielen, deren Beachtlichkeit nicht auf Knappheitslagen begrenzt war und die auf neuartige Gefährdungslagen eingestellt werden können. Der Abbau oder gar Wegfall der Knappheit der Verbreitungsmöglichkeiten ändert nichts an der fortbestehenden Maßgeblichkeit verfassungsrechtlicher Ziele der Rundfunkregulierung, kann aber den möglichen Weg der Zielerreichung beeinflussen. Neuartige Gefährdungen können darüber hinaus neue Wege der Zielverwirklichung erfordern.

11. Das Recht verfolgt unterschiedliche Ziele bei Schrankengesetzen einerseits (Art. 5 Abs. 2 GG) und Medien-Ausgestaltungsgesetzen andererseits (Art. 5 Abs. 1 Satz 2 GG). Die für die Funktionsweise der dualen Rundfunkordnung maßgebenden Ausgestaltungsgesetze müssen sich an ihrem Beitrag zur Sicherung der Funktionsfähigkeit der Medienordnung rechtfertigen lassen. Die Begriffe „kommunikative Vielfalt“ und „kommunikative Chancengerechtigkeit" bezeichnen anerkannte - im Einzelnen näher zu operationalisierende - Bündel von Zielen. Da ein zentraler Bezugspunkt der Rundfunkfreiheit die Meinungsbildungsfreiheit der Rezipienten ist, müssen rundfunkrechtliche Regeln auch auf die kommunikative Kompetenz der Bürgerinnen und Bürger abgestimmt sein.

12. Der Aufbruch in die Multimedia-Welt schafft keine Rechtfertigung, das Niveau der Zielerwartungen zu verringern. Im Gegenteil könnte er als Chance genutzt werden, dieses Niveau angesichts der neuen Möglichkeiten sogar höher als bisher anzusetzen. Jedenfalls ist es eine Herausforderung der Wissens- und Informationsgesellschaft, zumindest an den tradierten normativen Werten einer freiheitlichen Gesellschaft, so auch an denen der Emanzipation der Bürgerinnen und Bürger und des Schutzes vor Manipulation, festzuhalten und in der gegenwärtigen Zeit des multimedialen Umbruchs das gesellschaftlich verfügbare Potenzial an Werten, Informationen und Ideen möglichst weitgehend zugänglich zu machen und für möglichst viele nutzbar werden zu lassen. Es sollte auch denjenigen zugute kommen, denen dies bisher aufgrund ihrer Lebenssituation oder begrenzter persönlicher Kompetenzen erschwert war.

13. Die empirischen Anzeichen deuten demgegenüber eher in die Richtung, dass diese Ziele nicht vorrangiger Bezugspunkt der Entwicklung sind. In erster Linie werden ökonomische Entfaltungsinteressen befriedigt; Manipulationsrisiken nehmen zu; die Kluften in den soziokulturellen, finanziellen und technologischen Zugangsmöglichkeiten werden eher größer, also z. B. Ungleichheiten eher verstärkt als abgebaut. Daraus könnte eine neue soziale Frage, die der Informations- und Wissensgesellschaft, entstehen.

\section{Regelungsziele und Risiken ihrer Verfehlung}

14. Die Ausgestaltung der Rundfunkordnung muss dem Ziel „gleichgewichtiger Vielfalt" unter Vermeidung der Risiken von Fehlentwicklungen dienen. Hoheitliche Rundfunkregulierung ist als Mittel gerechtfertigt, soweit alleinige Selbstregulierung - d. h. in einer privatwirtschaftlichen Ordnung regelhaft Marktregulierung - zu verfassungsrechtlich gewichtigen Fehlentwicklungen (Marktversagen) führen würde, die nach der Einschätzung des Gesetzgebers durch Regulierung vermieden oder doch gemildert werden können. Nicht zu übersehen sind aber auch Risiken des Regulierungsversagens; diese dürfen nicht gewichtiger sein, sondern sollten möglichst im Vergleich zu denen des Marktversagens als geringer zu bewerten sein. 
15. Medienökonomische Analysen unter besonderer Berücksichtigung struktureller Besonderheiten der Medienmärkte zeigen Defizite einer alleinigen Marktsteuerung. Insbesondere die Theorien, die sich mit der Produktion meritorischer Güter, mit externen Effekten und mit der Netzwerkökonomie befassen, können solche Risiken auch theoretisch plausibilisieren und zeigen, dass im Medien- und Informationsmarkt strukturell besondere Risiken angelegt sind. Der aktuelle Umbruch der Medienlandschaft indiziert besondere Macht- und Konzentrationspotenziale sowie Missbrauchsrisiken, insbesondere bei der Zugangsfilterung. Die Palette möglicher Risiken ist größer und vielschichtiger, als das Bundesverfassungsgericht in seiner bisherigen Rechtsprechung berücksichtigen konnte.

16. Da es schon medienrechtliche Regulierungen gibt, ist insbesondere zu fragen, ob sie ausreichen bzw. zur Fehlsteuerung führen. Gegenstand der medienverfassungsrechtlichen Beurteilung ist nicht die Chance für die Verwirklichung wirtschaftlicher Interessen - etwa der gewinnmaximalen Teilhabe an der Wertschöpfung im MultimediaMarkt -, sondern die auf publizistischem Wettbewerb aufbauende Funktionsfähigkeit der Medienordnung, die der Sicherung der Medienfreiheit auch und in erster Linie als Meinungsbildungsfreiheit der Bürgerinnen und Bürger dient.

17. Die weitgehende Entkoppelung der verschiedenen freiheitsrelevanten Ebenen von Produktion, Veranstaltung i. e. S. und Verbreitung und deren weitere Ausdifferenzierung (s. Punkt 6 und 72) führen $\mathrm{zu}$ ebenenspezifischen Fragen nach Risiken und entsprechenden Regelungsbedarfen und Regelungsmöglichkeiten und nach Möglichkeiten der kooperativen Verkoppelung. Auf jeder der Ebenen ist zu fragen, wieweit die Ziele des Art. 5 Abs. 1 Satz 2 GG durch marktmäßige Organisation erreicht werden können oder wieweit ergänzend oder ersetzend besondere Ausgestaltungen erforderlich sind.

\section{Gefährdungen der Zugangschancengerechtigkeit}

18. Aktuelle medienpolitische Vorschläge befassen sich zwar auch, aber nur in eingeengter Hinsicht, mit den aktuell dringend gewordenen Problemen der Zugangsfilterung und damit dem Risiko der Verfehlung der Zugangschancengerechtigkeit. Wichtige normative Zielebenen verweisen auf die Notwendigkeit von Sicherungen für

- die Zugangschancengerechtigkeit für Kommunikatoren, insbesondere im Sinne eines Zugangs zu Produktionsfaktoren, zu Verbreitungs- und Vermarktungsmöglichkeiten;

- die Empfangschancengerechtigkeit für Nutzer, insbesondere im Sinne einer Zugänglichkeit der Netzinfrastruktur, der Verfügbarkeit nicht nur quantitativ, sondern auch qualitativ den Bedürfnissen angemessener, auf die gesellschaftlichen Möglichkeiten abgestimmter Inhaltsangebote;

- eine manipulationsfreie (-arme) Rezeptionssituation und

- die Zugänglichkeit in einer auf die kommunikative Kompetenz der Rezipienten abgestimmten, sie gegebenenfalls fördernden Weise.

19. Zugangsprobleme gibt es in verschiedenen, miteinander verkoppelten Dimensionen, so insbesondere als (vorrangig)

- vertriebsbezogene Zugangsprobleme;

- angebotsbezogene Zugangsprobleme;

- rezipientenbezogene Zugangsprobleme. 
20. Gegenwärtig sind die Probleme des Zugangs zu Verbreitungsinfrastrukturen von besonderer Relevanz, da dort Weichen gestellt und Strukturen eingerichtet werden, die in Zukunft nur schwer korrigierbar sein dürften. Problematische Zugangshürden können insbesondere in folgenden Kontexten errichtet werden:

- Setzung von Konditionen für den Zugang zur Verbreitung von Kommunikationsdiensten;

- Multiplexing;

- Programmpaketvermarktung;

- Navigationssysteme.

21. Nachhaltige Vermachtungsrisiken gibt es auch mit dem Blick auf die Zugänglichkeit der Rundfunkveranstalter zu Produktionsressourcen und Programmrechten.

22. Die zurzeit beobachtbare Tendenz zur Verkoppelung von Marktmacht in unterschiedlichen Sektoren der Medienmärkte verändert die Kommunikationsverhältnisse nachhaltig, ohne dass es schon angemessene regulative Gegenvorkehrungen für den Fall der Gefährdung der Funktionsfähigkeit der Medienordnung gäbe.

\section{Medienregulierung als hoheitlich regulierte Selbstregulierung}

23. Rundfunkregulierung gilt zwar einem besonderen Gegenstandsbereich mit spezifischen Regulierungsaufgaben, kann aber grundsätzlich auf die gleichen Regulierungskonzepte und -instrumente zugreifen, die in anderen Zusammenhängen für hoheitliche Regulierungen entwickelt wurden. Gegenwärtig werden die Aufgaben und die Verantwortungsteilung zwischen Hoheitsträgern (Staat) und Gesellschaft in vielen Bereichen neu konzipiert. Dazu gehören die weltweiten, auch im EG-Bereich forcierten, Bemühungen, möglichst weitgehend auf Selbstregulierung zu vertrauen. Selbstregulierung gibt es nicht nur in Form der Marktregulierung.

24. Soweit der Staat - wie aufgrund des Art. 5 Abs. 1 Satz 2 GG - in Bereichen mit Selbstregulierung weiter Verantwortung für die Verwirklichung bestimmter Ziele oder die Wahrnehmung bestimmter Aufgaben trägt, muss er einen regulativen Rahmen für die Selbstregulierung bereitstellen (hoheitlich regulierte gesellschaftliche Selbstregulierung). Ergänzend kann es erforderlich sein, seine Auffangverantwortung insbesondere zur Abfederung negativer Folgewirkungen der Selbstregulierung zu nutzen.

25. Die Aufgaben der Sicherung der Freiheitlichkeit der Kommunikation und das damit gekoppelte Gebot der Staatsferne bzw. Staatsfreiheit haben dazu geführt, dass der Medienbereich seit jeher auf Selbstregulierung, und zwar auf je unterschiedliche Typen der Selbstregulierung, ausgerichtet ist, dass der Gewährleistungsauftrag des Staates aber eine regulative Umhegung erlaubt und fordert, soweit sonst publizistische Gemeinwohlbelange nicht hinreichend berücksichtigt werden.

26. In heuristischer Absicht sollen mit dem Begriff der rundfunkrechtlichen „Steuerung" Tätigkeiten von Hoheitsträgern bezeichnet werden, mit denen sie auf die Akteure im Rundfunkbereich einwirken, um dem Gewährleistungsauftrag des Art. 5 Abs. 1 Satz 2 GG nachzukommen. Dass sie dabei nur in den Grenzen der Verfassung, also insbesondere unter Ausschluss programminhaltlicher Lenkung, handeln, muss stets gesichert bleiben.

27. Sowohl die Steuerung komplexer sozialer Systeme als auch des Verhaltens Einzelner ist schwierig. Deterministische, auf lineare Kausalitäten ausgerichtete Steuerungskon- 
zepte sind meist untauglich; unter den gegenwärtigen Ungewissheitsbedingungen und angesichts der vielfältigen Vernetzungen sind insbesondere verschlungene, nicht leicht nachvollziehbare Wirkungspfade und dynamische Rückkoppelungen einzukalkulieren, so dass es sich empfiehlt, entsprechend voraussetzungsvolle Steuerungskonzepte zu nutzen.

28. Typisch für moderne Medienregulierung ist eine Steuerung unter Nutzung von rechtlichen Rahmen- und Strukturvorgaben, die durch einzelne Verhaltensregeln ergänzt werden. Medienaufsicht unterstützt die Struktursteuerung (z. B. durch begrenzte Programminhaltsbindungen, Werberestriktion etc.) und ermöglicht die Korrektur von Fehlverhalten im Einzelfall.

\section{Schwierigkeiten erfolgreicher Regulierung}

29. Rechtliche Einwirkungen auf ein gesellschaftliches Feld (inputs) können - neben Folgenlosigkeit - Folgen verschiedener Art bewirken. Von der Veranlassung konkreten Verhaltens (output) sind die Wirkungen auf die konkret Betroffenen (impact) und davon die Auswirkungen in den betreffenden gesellschaftlichen Bereichen (outcome) zu unterscheiden.

30. Welche rechtlichen Instrumente zum Erfolg führen, lässt sich nicht gegenstandsneutral und abstrakt bestimmen. Steuerungserfolge werden maßgebend durch die in dem Regelungsfeld bestimmenden Handlungsrationalitäten („Eigenlogiken“) beeinflusst. Am ehesten werden rechtliche Vorgaben befolgt, wenn sie Optionen bereitstellen, die ein Verhalten erlauben, das mit den Eigeninteressen der Betroffenen kompatibel ist oder das ihnen besondere Vorteile verspricht. Diese Eigeninteressen sind allerdings häufig nicht bei allen Betroffenen identisch, so dass die Interessenheterogenität der verschiedenen Akteure berücksichtigt werden muss und gegebenenfalls auch für die Steuerung (strategisch) genutzt werden kann.

31. Rechtliche Steuerung gegen die Interessen der Betroffenen führt zu Ausweichreaktionen und ist nur ausnahmsweise - etwa unter Einsatz von Repression - erfolgreich. Repression aber ist in einer auf Selbstregulierung aufbauenden Ordnung ein Fremdkörper und kann daher nur ausnahmsweise Erfolg versprechend eingesetzt werden.

32. Soweit die Verfolgung der Eigeninteressen nicht zur Befriedigung von Gemeinwohlinteressen ausreicht, sollte darauf hingewirkt werden, den Betroffenen einen Verhaltenskorridor zu eröffnen, der es ihnen zwar ermöglicht, aber auch nur erlaubt, Optionen zu wählen, die zugleich gemeinwohlverträglich sind. Zielverwirklichung durch Kooperation der hoheitlichen Verantwortungsträger mit den Betroffenen ist ein häufig gewählter Weg in einer im Wesentlichen auf Selbstregulierung beruhenden rechtlichen Ordnung.

33. Die Erfahrungen mit Medienrecht und Medienaufsicht - in Diskussionen meist konzentriert und reduziert auf Rundfunkaufsicht - geben weltweit viele Anwendungsbeispiele für die Schwierigkeiten der hoheitlichen Steuerung und damit für die Richtigkeit der soeben allgemein formulierten Thesen. Aufsichtliche Einwirkungen auf den privaten Rundfunk waren zwar nicht durchgängig folgenlos; sie haben insbesondere zu einer relativen Ordnung der Entwicklung des Rundfunkwesens beigetragen. Aufsicht war aber umso schwieriger und zum Teil folgenloser, je stärker die Aufsichtsziele und -instrumente mit den Orientierungen der Unternehmen am Markterfolg kollidierten (so z. B. bei programminhaltlichen Vorgaben oder bei der Konzentrationsbekämpfung). 
Allerdings gab es auch erhebliche regulative Einwirkungen, die den Beaufsichtigten (oder einem Teil von ihnen) zugute kamen oder sie zumindest nicht behinderten.

34. Es gibt keine Anhaltspunkte für die Annahme, dass hoheitliche Regulierung und Aufsicht unter den neuen Bedingungen der Multimedia-Wirtschaft leichter fallen bzw. erfolgreicher sein werden als die traditionelle Rundfunkaufsicht. Die Ausdifferenzierung der verschiedenen Wertschöpfungsakte und die Möglichkeiten zu ihrer unterschiedlichen Kombination werden Aufsicht ebenso erschweren wie die zunehmende Internationalisierung.

35. Auf Befunde nachhaltiger Nichtbefolgung bzw. des Unterlaufens aufsichtlicher Maßnahmen hat die Medienaufsicht häufig mit einer Zurücknahme der Anforderungen und der Gesetzgeber mit einer Modifikation der Regulierungsziele und/oder Abschwächung der Aufsichtsinstrumente - also durch einen problematischen normativen Verzicht - reagiert.

36. Die Erfüllung des gesetzlichen Gewährleistungsauftrags des Art. 5 Abs. 1 Satz 2 GG bemisst sich am impact (an der Art des Gebrauchs der Rundfunkfreiheit durch die Grundrechtsbegünstigten) und am outcome (insbesondere an der Funktionsfähigkeit der Medienordnung als Teil der Gesellschaftsordnung). Rechtliche Maßnahmen sind meist so konstruiert, dass sie auf einen bestimmten output hinzielen, von dem angenommen wird, dass impact und outcome in der erwünschten Weise beeinflusst werden. Die auf die Veranstaltung von Rundfunk ausgerichtete Steuerung hat es allerdings schwer, erwünschte outputs (und in der Folge den angestrebten impact und outcome) zu bewirken.

\section{Zusammenspiel von Medienrecht und anderen Teilen der Rechtsordnung}

37. Ein Teil der Regulierungsprobleme lässt sich durch Rückgriff auf sonstige Teile der Rechtsordnung (Telekommunikations-, Zivil-, Wirtschafts-, Urheberrecht u. a.) bewältigen, die zusammen mit Medienrecht als wechselseitig nutzbare Teil-Auffangordnungen verstanden werden können. Die rechtlichen Teilgebiete folgen aber eigenen Zielen und Rationalitäten und sind nicht geeignet, den Bedarf zur Sicherung der Funktionsfähigkeit der Rundfunkordnung so zu befriedigen, dass auf rundfunkspezifische Regulierung verzichtet werden könnte.

38. Der Vorschlag, den ordnungspolitischen Rahmen für Rundfunk zu ändern und Rundfunkrecht in dem allgemeinen Wirtschaftsrecht aufgehen zu lassen, ist mit den Grundprinzipien der deutschen dualen Rundfunkordnung nicht vereinbar, weil er darauf zielt, den ökonomischen Wettbewerb zum zentralen Steuerungsmodus im Programmbereich zu erheben. Auf diesem Steuerungsmodus aber baut allgemeines Wirtschaftsrecht auf. Es wäre ein Strukturwiderspruch, öffentlich-rechtlichen Rundfunk einerseits von ökonomischen Imperativen freizustellen (Gemeinwirtschaftlichkeit) und ihn im Programmverhalten zugleich allein oder auch nur vorrangig ökonomischen Steuerungsimpulsen auszusetzen.

39. Für die Regulierung privatwirtschaftlichen Rundfunks scheidet ein durch spezifische rundfunkrechtliche Bindungen modifiziertes Wirtschaftsrecht allerdings nicht von vornherein aus. Die gebotene rundfunkspezifische Modifikation könnte allerdings zum Funktionswandel des Wirtschaftsrechts und der wirtschaftsrechtlichen Aufsicht führen und damit den Charakter des Wirtschaftsrechts als eines allgemeinen - also im Regelfall 
losgelöst von den spezifischen Strukturen und Leistungsmerkmalen des jeweiligen Marktes wirkenden - Rechtsrahmens unterminieren.

40. Eine entsprechende wirtschaftsrechtliche Regulierung könnte aus Gründen fehlender Gesetzgebungskompetenz des Bundes nicht als Ausgestaltungsgesetz im Sinne des Art. 5 Abs. 1 Satz 2 GG ergehen; als Schrankengesetz im Sinne des Art. 5 Abs. 2 GG aber könnte sie nicht speziell auf die Sicherung der Funktionsfähigkeit der Medienordnung ausgerichtet sein. Inhaltlich müsste eine rein wirtschaftsrechtliche Steuerung zur Notwendigkeit anderweitiger Absicherungen der Funktionsfähigkeit der Medienordnung führen. Gemäß der Logik der dualen Rundfunkordnung, nämlich wegen des normativen "Scharniers" bei der Vielfaltssicherung (s. o. Punkt 2 und 3), käme als Alternative in Betracht, die Fähigkeit des öffentlich-rechtlichen Rundfunks zur Abfederung von Vielfaltsdefiziten privaten Rundfunks im Vergleich zum Status quo zu verstärken.

\section{Aufgabe fortwährender Überprüfung der Tauglichkeit der Regulierung beider Teile der dualen Rundfunkordnung}

41. Auch schon vorhandene Regulierungen gehören auf den Prüfstand der fortwährenden Funktionstauglichkeit. Dies gilt für die Regelungen beider Säulen der Rundfunkordnung sowie für deren Zusammenspiel. Die Fixierung der gegenwärtigen Diskussion fast nur auf öffentlich-rechtlichen Rundfunk bewirkt eine der dualen Ordnung nicht angemessene Schieflage.

42. Rundfunkgesetzgebung ist Gesetzgebung zur freiheitsermöglichenden Ausgestaltung der Rundfunkordnung. Sie ist daher auf die Funktionsfähigkeit dieser Rundfunkordnung bezogen. Es gibt selbstverständlich auch rechtliche Grenzen zulässiger Ausgestaltung. In dem durch sie markierten „Korridor“ geht es allerdings in erster Linie um Optimierung bei der Auswahl und Kombination von Gestaltungsoptionen. Deshalb hat die wissenschaftliche und praktische Diskussion um Rundfunkausgestaltung in vielem notwendig den Charakter einer medienpolitischen Diskussion.

43. In der aktuellen medienpolitischen Diskussion wird das Nebeneinander von öffentlich-rechtlichem und privatem Rundfunk als solches nicht zur Disposition gestellt. Die grundsätzliche Akzeptanz und wechselseitige Koexistenz beider Säulen sind gegenwärtig gesichert. Insofern besteht kein Anlass zur Umsteuerung.

\section{Insbesondere: Zum Funktionsauftrag öffentlich-rechtlichen Rundfunks}

44. Der Vorwurf einer Expansion öffentlich-rechtlichen Rundfunks ist der empirischen Prüfung zugänglich, diese bedarf dafür aber eines normativen Maßstabs. Eine Expansion durch Tätigkeiten außerhalb des gesetzlichen Auftrages wäre rechtswidrig, also korrekturbedürftiges Fehlverhalten. Nicht als Expansion, sondern als Erfüllung seines Entwicklungsauftrages einzuordnen wäre allerdings eine (aufgabengerechte) Reaktion des öffentlich-rechtlichen Rundfunks auf Marktveränderungen; sein Bemühen, in der publizistischen Konkurrenz mit privatem Rundfunk erfolgreich zu sein - z. B. durch entsprechende Ausdifferenzierung des Programmangebots -, ist der dualen Rundfunkordnung gemäß.

45. Begrenzungen des Programmauftrags öffentlich-rechtlichen Rundfunks sind verfassungsrechtlich nicht von vornherein ausgeschlossen. Programmbegrenzungen bedürfen wegen des Gebots der Staatsfreiheit auch bei gesetzgeberischem Handeln aber besonde- 
rer, insbesondere prozeduraler, Absicherungen und müssen inhaltlich an dem Ziel der Verbesserung der Funktionsfähigkeit der dualen Rundfunkordnung gerechtfertigt werden.

46. Der Vorschlag einer Bereichsabgrenzung öffentlich-rechtlichen Rundfunks durch Präzisierung eines „Funktionsauftrags“ hat viele Vorläufer, die darauf gezielt haben, das Betätigungsfeld öffentlich-rechtlichen Rundfunks zugunsten der Entfaltungschancen privaten Rundfunks zu begrenzen.

47. Der in dieser Diskussion zur Ersetzung des Begriffs der Grundversorgung neu eingeführte Begriff des Funktionsauftrags führt nicht, jedenfalls nicht notwendig zur Begrenzung des Aufgabenfeldes des öffentlich-rechtlichen Rundfunks. Schon der - in der Tat missverständliche - Vorgängerbegriff der „Grundversorgung“ war ein zukunftsoffen formulierter Funktionsbegriff, der die Entwicklungsdynamik aufgreifen wollte und eine Bereichsabgrenzung oder gar Marginalisierung des öffentlich-rechtlichen Rundfunks gerade ausschließen sollte.

48. Richtig - nämlich entwicklungsoffen - verstanden ist der Begriff „Funktionsauftrag“ besser als der bisher übliche des „Programmauftrags“ geeignet, die verschiedenen Teilaufgaben des öffentlich-rechtlichen Rundfunks funktionsbezogen zu bündeln, die zwar stets auf das Programm bezogen sein müssen, sich aber in der ausdifferenzierten dynamischen Medienwelt im Interesse der Funktionsfähigkeit der Rundfunkordnung auch in die der Veranstaltung i. e. S. vor-, neben- und nachgelagerten Bereiche erstrecken.

49. Weder mit der (richtig verstandenen) Komplementärfunktion noch mit der Integrationsfunktion lässt sich rechtfertigen, öffentlich-rechtlichen Rundfunk auf Vollprogramme und bestimmte (nicht massenattraktive) Spartenprogramme zu begrenzen oder zielgruppenbezogene Programme grundsätzlich auszuschließen. Ebenso gibt es keine Rechtfertigung, ihm massenattraktive Unterhaltung vorzuenthalten. Stets, also auch im Bereich massenattraktiver Programme, bedarf es Sicherungen einer meritorischen Qualität der Programmangebote.

50. Dies gilt auch für den Fall weiterer Fragmentierung der Rundfunkprogramme, etwa im Sinne einer Zunahme von Sparten- und Zielgruppenprogrammen. An solchen Entwicklungen teilzunehmen, ist dem öffentlich-rechtlichen Rundfunk nicht grundsätzlich verwehrt. Die Sicherung einer besonderen Public-Service-Qualität in dem betreffenden Angebot (nicht zwingend in jeder einzelnen Sendung) ist aber Bedingung der Veranstaltung solcher Programme.

51. Eine Schutzzone privaten Rundfunks vor publizistischem Wettbewerb durch öffentlich-rechtlichen Rundfunk wäre in der dualen Rundfunkordnung mit der Idee der Vielfaltssicherung durch Angebote von Trägern zweier unterschiedlich strukturierter und orientierter Teilordnungen unvereinbar. Da alle Programmsegmente für die Meinungsbildungsfreiheit der Bürgerinnen und Bürger relevant sind oder doch sein können, muss der publizistische Wettbewerb als Modus der Verwirklichung medienverfassungsrechtlicher Ziele für alle funktional äquivalenten Programmangebote nutzbar sein. Ein funktionierender publizistischer Wettbewerb setzt also voraus, dass beide Säulen alle Zuschauer- bzw. Hörerschaften unter Berücksichtigung ihrer Kommunikationsbedürfnisse und Rezeptionsgewohnheiten bedienen dürfen (nicht müssen) und versuchen, im Rahmen ihres spezifischen Auftrags die jeweils besseren Rezeptionschancen zu haben $-\mathrm{z}$. B. über unterschiedliche Präsentationsformen, programmliche Schwerpunkte, berücksichtigte Vielfaltsdimensionen oder durch sonstige inhaltliche Qualitäten. 


\section{Entwicklungsmöglichkeiten}

52. Privatem wie öffentlich-rechtlichem Rundfunk steht nach Maßgabe der jeweils für sie geltenden Gesetze der Zugang zu allen Verbreitungstechnologien offen, auch zum Internet.

53. Bei der Internet-Nutzung sind dabei unterschiedliche Betätigungsfelder zu unterscheiden.

(1) Die Nutzung des Internet als Medium für ergänzende Informationen zum üblichen Rundfunkprogramm (Annexdienste).

(2) Die Nutzung des Internet zur Verbreitung von Rundfunkprogrammen, die auch über andere Verbreitungstechnologien verbreitet werden (Erweiterung der technischen Plattform).

(3) Die Nutzung des Internet zur Verbreitung neuartiger, speziell auf das Internet abgestimmter Programminhalte.

54. Für privatwirtschaftlichen Rundfunk hat der Mediendienste-Staatsvertrag (MDStV) eine Einengung der Geltungskraft des Rundfunkstaatsvertrages (RStV) gebracht: Die Anwendung des Landesrundfunkrechts auf Mediendienste ohne Charakter der „Darbietung“ (ohne stärkere Meinungsrelevanz bzw. ohne besondere kommunikative Wirkungskraft) ist in der Folge ausgeschlossen.

55. Der 4. Rundfunkänderungsstaatsvertrag betrifft und begrenzt die Annextätigkeiten des öffentlich-rechtlichen Rundfunks, schließt aber die Nutzung des Internet zur (zusätzlichen) Verbreitung ohnehin veranstalteter Rundfunkprogramme ebenso wenig aus wie die Nutzung des Internet für neuartige Rundfunkdienste. Weder der MDStV noch der RStV regelt bzw. begrenzt die Nutzung des Internet durch öffentlich-rechtlichen Rundfunk. Entscheidend für sein Tätigkeitsfeld ist vielmehr, ob die Rundfunkgesetze die Internet-Nutzung ausschließen. Dies ist eine Frage der Auslegung der Normen über den Funktionsauftrag öffentlich-rechtlichen Rundfunks.

56. Der Vorschlag, privatem Rundfunk die Finanzierung über Werbung und Sponsoring vorzubehalten, d. h. sie öffentlich-rechtlichem Rundfunk zu verwehren, würde der Idee struktureller Diversifikation in besonderem Maße entsprechen; seine Umsetzung ist allerdings verfassungsrechtlich nicht geboten. Sie bedürfte begleitender Vorkehrungen zur Erfüllung des Gebots funktionsgerechter Finanzierung.

57. Pay-TV durch öffentlich-rechtliche Rundfunkanstalten wäre zwar nicht von Verfassung wegen ausgeschlossen, seine Einführung wäre aber in medienpolitischer Hinsicht für öffentlich-rechtlichen Rundfunk gegenwärtig riskant.

58. Eine Finanzierung öffentlich-rechtlichen Rundfunks aus Steuermitteln wäre verfassungsrechtlich nur zulässig, wenn besondere - insbesondere prozedurale - Sicherungen der Staatsunabhängigkeit eingeführt würden.

59. Eine Neuordnung der Medienaufsicht, insbesondere der Aufsicht über privaten Rundfunk, kann sinnvollerweise nicht abgelöst von der Art der Regulierung erfolgen. Viele der aktuell diskutierten Vorschläge über die Konzentration bzw. Veränderung von Aufsicht stehen in untrennbarem Zusammenhang mit dem Regulierungskonzept selbst, ohne dass sie aber entsprechend weiträumig und tief konzipiert werden. Die Diskussion ist daher unzuträglich verkürzt. 


\section{Selbstregulierung und Aufsicht beim privaten Rundfunk}

60. In der privatwirtschaftlichen Säule der Rundfunkordnung ist maßgebendes Ordnungsprinzip die über den Markt erfolgende, ökonomisch determinierte Selbstregulierung. Mit ihr sind in der Rechtsordnung die Grundelemente von Privatwirtschaftlichkeit und -rechtlichkeit gekoppelt, ohne dass dies einer spezifischen Regulierung bedürfte. Das Privatrecht schafft den institutionellen Rahmen für Privatautonomie. Autonom bestimmtes und verwaltetes Eigeninteresse wird zur maßgebenden Steuerungsressource; Privateigentum und Wettbewerb sind konstituierende Elemente. Medienrecht ergänzt dies durch eine an den Zielvorgaben des Art. 5 GG orientierte regulative Umhegung privatautonomen Verhaltens (regulierte Selbstregulierung).

61. Entfaltung am Medienmarkt unter Nutzung seiner Eigenrationalitäten heißt primäre Orientierung am wirtschaftlichen Erfolg; erlaubt und „normal“ ist die Verfolgung eines Ertrags- und Gewinninteresses (abgesichert in Art. 12, 14, 2 Abs. 1 GG). „Verdienen“ (im Eigeninteresse), nicht etwa „Dienen“ (am Gemeinwohl) ist legitimerweise die Leitlinie der Marktorientierung. Rundfunkrecht muss aber wegen der Vorgaben des Art. 5 Abs. 1 Satz 2 GG um praktische Konkordanz von „Verdienen“ und „Dienen“ bemüht sein. Die Teilhabe privaten Rundfunks am publizistischen Wettbewerb erfolgt nach Maßgabe des wirtschaftlichen Wettbewerbs. In einer dualen Ordnung ist dies verfassungsrechtlich unbedenklich, weil (und solange) die möglicherweise so nicht zu erfüllenden medienverfassungsrechtlichen Vielfaltsanforderungen jedenfalls durch den öffentlich-rechtlichen Rundfunk bedient werden können (s. o. Punkt 3).

62. Selbstregulierung bildet sich in der privatwirtschaftlichen Rundfunkordnung auf verschiedenen Ebenen ab, so als unternehmensinterne, aber auch als marktbezogene Selbstregulierung. Auch die verschiedenen Selbstkontrolleinrichtungen der Medienwirtschaft sind Erscheinungsformen von Selbstregulierung, die aber auf enge Aufgabenfelder begrenzt sind. Sie bewirken eine (begrenzte) Entlastung der hoheitlichen Medienaufsicht.

63. Die Rundfunkordnung ist umso leistungsfähiger konzipiert, je weniger die Rundfunkaufsicht in konkreten Einzelfällen zur Gegensteuerung benötigt wird, d. h. je stärker die Selbstregulierung der Rundfunkwirtschaft zur Erzielung auch der erwünschten Gemeinwohlwirkungen beiträgt. Rundfunkaufsicht kann aber unentbehrlich zur gemeinwohlorientierten Regulierung dieser Selbstregulierung sein.

64. Die hoheitliche Rundfunkaufsicht durch Landesmedienanstalten als grundrechtssichernden Anstalten hat im Laufe der Zeit in Verarbeitung der Erfahrungen mit praktizierter Medienaufsicht (s. o. Punkt 33) einen Wandel durchgemacht. Ein von der Arbeitsgemeinschaft der Landesmedienanstalten im Jahre 1999 ausgearbeitetes Positionspapier dokumentiert das Bemühen, die Verantwortung für konkrete Ergebnisse und Wirkungen von Aufsicht (die sog. Erfüllungsverantwortung) zurückzunehmen und sich verstärkt auf Setzung eines Rahmens und von Spielregeln zu konzentrieren, vor allem aber konzipierend, beratend und koordinierend tätig zu werden (im Zuge der sog. Gewährleistungsverantwortung). Dies wird durch eine als Auffangverantwortung zu verstehende Missbrauchsaufsicht und Sorge für den Schutz von Minderheiten ergänzt. Solche Veränderungen entsprechen einem weltweit beobachtbaren Trend der Regulierung.

65. Hoheitliche Medienaufsicht ist in vielem auf die Mitwirkungsbereitschaft der Beaufsichtigten angewiesen (s. o. Punkt 32). Die Kooperation kann die Aufsichtsträger in den Augen externer Beobachter diskreditieren (Eindruck von Kollusion u. ä.); auch be- 
stehen Risiken einer unzuträglichen Nähe zu den Regulierten (Distanzverlust), der Übernahme ihrer Perspektiven oder gar der Verstrickung in deren Handlungsrahmen (capture).

\section{Selbstregulierung und Aufsicht beim öffentlich-rechtlichen Rundfunk}

66. In der öffentlich-rechtlichen Säule ist maßgebendes Ordnungsprinzip ebenfalls die (regulierte) Selbstregulierung, aber in anderer Weise als beim privaten Rundfunk. Als rechtliche Konstrukte des Staates mit einem spezifisch definierten Aufgabenfeld ist den Rundfunkanstalten nach Maßgabe der vom Gesetzgeber geschaffenen Strukturen rechtliche Autonomie gewährt. Eine Orientierung am Gewinninteresse ist ihnen untersagt. Rundfunkfreiheit darf nicht als „verdienende“ Freiheit genutzt werden. Das verfassungsrechtliche Gebot funktionsgerechter Finanzierung des öffentlich-rechtlichen Rundfunks soll sichern, dass die Einhaltung dieser Vorgabe möglich ist.

67. Selbstregulierung wirkt sich auch beim öffentlich-rechtlichen Rundfunk auf verschiedenen Ebenen aus. Ein wichtiges Feld ist organisationsinterne Selbstregulierung (auch durch plural zusammengesetzte Organe etc.), die durch selbstregulative Vorkehrungen für Effizienz (etwa durch Einführung neuer Managementstrukturen, Budgetierung, Controlling u. ä.) ergänzt wird.

68. Hinzu kommt in programmlicher Hinsicht eine der Public-Service-Idee verpflichtete Selbstregulierung. Die Maßstäbe dafür sind in professioneller Eigenverantwortung zu konkretisieren. Interner Anstaltsaufbau und (vorrangige) Gebührenfinanzierung sind strukturelle Vorkehrungen dafür, dass die professionelle Verantwortung der im Programmbereich Tätigen sich in Distanzierung vom ökonomischen Markt folgenreich an Kriterien publizistischer Relevanz orientiert.

69. Die aktuellen Versuche der Rundfunkanstalten zur Operationalisierung der PublicService-Orientierung und der Herausarbeitung eines spezifischen Programmprofils und damit zur ausdrücklichen Entwicklung von Maßstäben der Programmqualität sowie zum Aufbau eines Qualitätsmanagements und Qualitätscontrolling zeigen Möglichkeiten selbstregulativer Reaktion auf sich verändernde Rahmenbedingungen. Sie sind auch in ausgelagerte, programmrelevante Bereiche hin zu erstrecken.

70. Die vorrangig binnenorganisatorisch (plural) eingerichtete Rundfunkaufsicht ist ein Teilelement selbstregulativer Organisation, die durch die regulativen Rahmenvorgaben des Rundfunkrechts vorstrukturiert worden ist und deren Funktionieren durch die (verfassungsrechtlich notwendig begrenzte) staatliche Rechtsaufsicht überwacht wird. Die hoheitliche Rechnungsprüfung durch Rechnungshöfe und die mit der Gebührenfinanzierung gekoppelte externe Rechenschaftspflicht gegenüber der Kommission zur Ermittlung des Finanzbedarfs (KEF) und dem Parlament sind weitere regulative Umhegungen der Selbstregulierung.

71. Für eine auf Public Service ausgerichtete Einrichtung ist Rechenschaft gegenüber der Allgemeinheit (Öffentlichkeit) eine besonders wichtige Bezugsgröße der Legitimation (public accountability). Deswegen gewinnt Transparenz große normative Bedeutung für das Handeln der Rundfunkanstalten. Selbstregulative Prozesse können durch verstärkten Dialog mit der Öffentlichkeit bereichert werden, so z. B. durch deren verstärkte Einbeziehung in die Erarbeitung von Programmprofilen und das Qualitätsmanagement, durch eingehendere Informationen über Programmentwicklungsplanungen bis hin zur öffentlichen Konkretisierung von Zielvorgaben. 


\section{Die Zuordnung der beiden Säulen der Rundfunkordnung}

72. Für die Zuordnung der beiden Säulen gibt es - trotz anfänglichen Fehlens eines konzeptionellen Designs (s. o. Punkt 1) - einige typische Konstruktionsprinzipien als Konkretisierungen der Grundidee struktureller Diversifikation:

- die unterschiedliche Einstellung zum Prinzip Wettbewerb (publizistischer/ökonomischer Wettbewerb);

- damit verbunden die Anerkennung je unterschiedlicher Eigenrationalitäten (insbesondere privatwirtschaftliches Ertrags- und Gewinnmotiv einerseits und gemeinwirtschaftlich fundierte Gemeinwohlorientierung andererseits);

- unterschiedliche, auch unterschiedlich intensive Programmbindungen;

- unterschiedliche Verfahren der Zuteilung technischer Ressourcen, insbesondere der Übertragungskapazitäten;

- unterschiedliche Regeln über den jeweils bevorzugten Zugriff auf unterschiedliche finanzielle Ressourcen (Werbung einerseits, Gebühren andererseits);

- unterschiedliche Freiheiten zur Betätigung in anderen Marktsegmenten.

Demgegenüber wird die Zuordnung der beiden Säulen nicht durch eine programmgegenständliche Bereichsabgrenzung oder einen grundsätzlichen Ausschluss bestimmter Verbreitungstechnologien für eine der Säulen vorgenommen (s. o. Punkt 47 ff., $51 \mathrm{ff}$.$) .$

73. Das Zusammenspiel der beiden Säulen der dualen Ordnung erlaubt es in vielen Situationen, hoheitliche Interventionen in den jeweiligen Säulen vorsichtiger, schonender einzusetzen, als wenn das Vertrauen auf die Funktionsfähigkeit der gesamten Rundfunkordnung allein auf eine Säule gestützt wäre. So dürfen Funktionserwartungen an die eine Säule zurückgeschraubt werden, wenn sie in der anderen Säule in abgesicherter Weise, insbesondere ohne funktionswidrige Belastungen, befriedigt werden. Auch ist es möglich, normwidrige Funktionsdefizite in der jeweiligen Säule zu bekämpfen, ohne zugleich das duale System als solches zur Disposition stellen zu müssen. Strukturdualität ist insofern eine besondere Ausprägung des rechtsstaatlichen Grundsatzes möglichst schonender Intervention in Autonomiebereiche.

\section{Ausweitung des Prinzips struktureller Diversifikation}

74. Solange Tätigkeiten in den jetzt vor-, neben- und nachgelagerten Märkten noch zum selbstverständlichen Aufgabenfeld der öffentlich-rechtlichen Rundfunkanstalten selbst gehörten, wirkten sich deren Strukturprinzipien auch bei diesem Handeln aus. Die verstärkte Auslagerung von Tätigkeiten und die Abhängigkeit vom Erwerb benötigter Leistungen an den Medienmärkten haben zu einer Reduktion des Anwendungsbereichs gesicherter struktureller Diversifikation geführt und werden es vermutlich noch weiter tun. Daraus können Probleme der Funktionsfähigkeit der Medienordnung entstehen.

75. In der dualen Rundfunkordnung wirken sich die Probleme der Zugänglichkeit zu den Leistungen des Multimedia-Wertschöpfungsnetzwerks auf die privatwirtschaftliche und die öffentlich-rechtliche Säule in unterschiedlicher Weise aus.

a) Rechtlich sind die Unternehmen privaten Rundfunks nicht gehindert, sich indem gesamten Multimedia-Wertschöpfungsnetzwerk zu betätigen und damit - je 
nach den finanziellen u. ä. Möglichkeiten - aus eigener Kraft Zugangshürden zu überwinden.

b) Anders der öffentlich-rechtliche Rundfunk, der nicht ultra vires handeln darf. Allerdings sind die gegenwärtigen Aufgabennormen relativ weit gefasst und erlauben Tätigkeiten in den vor-, neben- und nachgelagerten Bereichen. Beschränkungen der Tätigkeit in vor-, neben- und nachgelagerten Bereichen sind für die Funktionsfähigkeit der Medienordnung nicht unproblematisch, da sie dem öffentlich-rechtlichen Rundfunk Möglichkeiten nehmen könnten, die vielen aktuellen und potenziellen Zugangshürden aus eigener Kraft (etwa durch eigene Betätigung) zu nehmen. Zum Ausgleich müssten in der Rechtsordnung andere (besondere) Vorkehrungen zur Sicherung der Zugänglichkeit geschaffen werden.

76. Die Vermachtungs- und Missbrauchsrisiken in den Multimedia-Netzwerken können es nahe legen, das Grundprinzip struktureller Diversifikation auch in andere Bereiche als die der Rundfunkveranstaltung i. e. S. zu erstrecken und neue Vorkehrungen zur Sicherung der Funktionsfähigkeit der Medienordnung, etwa in den Bereichen der Produktion, der Erstellung und des Betriebs der Kommunikationsinfrastruktur sowie der Dienste mit Zugangsfilterungsfunktion, vorzusehen.

77. $\iint 52,53 \mathrm{RStV} 1999$ dokumentieren den staatlichen Regelungsanspruch für ein Teilsegment und wollen insbesondere die Zugänglichkeit sichern. Dort werden in erster Linie „Spielregeln“ aufgestellt, z. B. für die Kabelverbreitung, aber auch für Zugangsdienste einschließlich der Navigatoren. Sollte der Gesetzgeber unter Nutzung seiner Einschätzungsprärogative zum Ergebnis kommen, dass die Vorkehrungen nicht reichen und dass auch eine Aufstockung der Verhaltensregeln und Sanktionen nicht effektiv oder gar kontraproduktiv ist, muss stattdessen gegebenenfalls auch für alternative Strukturen - etwa durch ergänzende gemeinwirtschaftliche Träger dieser Dienste - gesorgt werden. Der Gestaltungsspielraum des Gesetzgebers ist jedenfalls weit genug.

78. Die Grundidee struktureller Diversifikation ist nicht auf Rundfunkprogramme begrenzt, sondern kann auch auf andere kommunikative Dienste bezogen werden, die gemäß Art. 5 Abs. 1 Satz 2 GG regelbar sind und bei denen Regelungsbedarf besteht. Angesichts der Dynamik des Multimediabereichs und der Konvergenzprozesse können sich neue Regelungsbedarfe entwickeln, z. B. für den Teil der Mediendienste, die ohnehin verfassungsrechtlich vom Rundfunkbegriff erfasst werden bzw. in den Geltungsbereich des Art. 5 Abs. 1 Satz 2 GG fallen. Auch Dienste auf der Grenzlinie zwischen Massen- und Individualkommunikation können betroffen sein, sofern sie für die zukünftige Funktionsfähigkeit der Medienordnung wichtig sind. Art und Intensität möglicher Regulierung sind eigenständig zu bestimmen.

\section{Ausblick}

79. Das Bild von den zwei „Säulen“ der Rundfunkordnung beruht auf einer erheblich vereinfachten Realitätswahrnehmung. Es drückt nicht hinreichend aus, dass die Medienordnung aus einem komplizierten Gerüst mit unterschiedlichen und je unterschiedlich tragfähigen Pfeilern und unterschiedlich belastbaren Verstrebungen besteht. Auch gibt es in ihr faktisch vielfältige Kooperationsformen zwischen Akteuren beider "Säulen“ und zunehmend hybride Leistungsträger. Das Bild von den Säulen speichert gleichwohl in pragmatischer Absicht eine gedankliche Bündelung der strukturell gegensätzlichen 
Teilelemente der Medienordnung und kann insofern (wenn auch nur mit Vorbehalt) weiter genutzt werden.

80. Die privatwirtschaftliche Säule trifft auf keine besonderen rechtlichen Grenzen der Betätigung in den Segmenten der Multimedia-Märkte. Ihre Lebensfähigkeit bedarf keiner zusätzlichen Absicherung. Bestands- und Entwicklungsgarant privatwirtschaftlicher Medien ist der Markt. Überlebensgarantien gibt es im rechtlichen Rahmen nach Maßgabe der Marktgesetzlichkeiten, aber auch nur nach ihnen.

81. Demgegenüber hängt die Leistungsfähigkeit des öffentlich-rechtlichen Rundfunks von besonderen, rechtlich geschaffenen Handlungsvoraussetzungen ab, und zwar auch für die veranstaltungsbezogenen Tätigkeiten in den vor-, neben- und nachgelagerten Bereichen. Dementsprechend ist die Ausstrahlung der Bestands- und Entwicklungsgarantie in diese Bereiche hinein Grundlage der Zukunftsfähigkeit öffentlich-rechtlichen Rundfunks.

82. Eine zeitangemessene Bestimmung eines Funktionsauftrags öffentlich-rechtlichen Rundfunks reicht über den Bereich der Veranstaltung i. e. S. hinaus und muss insbesondere die vielfältigen Gefährdungen der Zugänglichkeit verarbeiten, die nicht nur im Produktionsbereich und beim Rechteerwerb bestehen können, sondern auch bei der Programmverbreitung und -rezeption (s. o. Punkt 18 - 22).

83. Im Einzelnen sind noch viele Fragen offen, so z. B. nach der für sonstige Tätigkeiten geforderten Nähe zur Programmveranstaltung i. e. S., nach den Grenzen der Kooperation mit Dritten, nach Regeln der Beteiligungsverwaltung, nach der Möglichkeit der Produktion auch für sonstige Medienunternehmen, nach der haushaltswirtschaftlichen Behandlung oder nach medienspezifischem Controlling. Wieweit selbstregulative Mechanismen ausreichen oder deren regulative Umhegung erforderlich ist, bedarf weiterer Abklärung.

84. Eine schleichende Ökonomisierung der fragmentierten Lebenswelten einer Rundfunkanstalt muss vermieden werden. Insofern wird auch zu klären sein, ob, wieweit und wie die rundfunkgesetzlich vorgesehenen strukturellen Sicherungen der Gemeinwohlorientierung - etwa die Zielvorgaben und die Verantwortlichkeit pluraler Gremien - in diese weiteren Bereiche hinein zu erstrecken sind. Aufgabe muss auch hier die Aktivierung von Selbstregulierungspotenzialen in einem die Public-Service-Orientierung der Rundfunkveranstaltung sichernden regulativen Rahmen sein.

85. Die Sicherung der Funktionsfähigkeit der Rundfunkordnung muss auf die Freiheit der Meinungsbildung der Rezipienten bezogen bleiben. Ihnen hilft es nicht, wenn es für ein Segment (für die Veranstaltung von Rundfunk i. e. S.) Sicherungen einer treuhänderischen Gemeinwohlbindung gibt, diese aber in anderen relevanten Segmenten (etwa bei der Produktion, der Verbreitung des Programms oder der Gestaltung der Rezeptionsbedingungen) unterlaufen, verdünnt oder verfälscht werden.

86. Es ist absehbar, dass die Medienordnung der näheren Zukunft in den wesentlichen Elementen privatwirtschaftlich gestaltet sein wird. Eine treuhänderische Orientierung an den Zielen kommunikativer Vielfalt, Chancengerechtigkeit und Kompetenz bleibt angesichts neuartiger Gefährdungen für mehrere Segmente der Medienbetätigung wichtig; gemeinwirtschaftliche Strukturen sind als Ergänzung der Marktstrukturen allem Anschein nach dafür unverzichtbar. Maßnahmen zur Sicherung der Freiheit der Kommunikation und Medien aller werden zukünftig vielleicht noch wichtiger als sie in der Vergangenheit waren und in der Gegenwart sind. Denn Vertrauen auf den Markt allein reicht angesichts seiner Funktionsdefizite nicht. Allerdings ist auch er ein wichtiger und in einer privatwirtschaftlichen Ordnung unverzichtbarer Modus der Selbstregulierung. 